\title{
In-vitro susceptibility of Giardia lamblia to albendazole, mebendazole and other chemotherapeutic agents
}

\author{
R. CEDILLO-RIVERA* and O. MUÑOZ \\ Unidad de Investigación Clinica en Enfermedades Infecciosas y Parasitarias, Hospital de Pediatría, \\ Centro Médico Nacional Siglo XXI, Instituto Mexicano del Seguro Social, México, D.F. 06725, México
}

\begin{abstract}
Summary. The susceptibility of a strain of Giardia lamblia to benzimidazole carbamates, 5nitroimidazoles, nitrofurans and other drugs was studied in vitro. Albendazole was the most active compound, with a $50 \%$ inhibitory concentration (IC50) of $0.01 \mathrm{mg} / \mathrm{L}$ and a minimal lethal concentration (MLC) of $<0.04 \mathrm{mg} / \mathrm{L}$; the IC50 of mebendazole was $0.06 \mathrm{mg} / \mathrm{L}$ and the MLC $<0.5 \mathrm{mg} / \mathrm{L}$. Among the 5-nitroimidazoles tested, ornidazole was the most effective (IC50 0.12 mg/L); tinidazole, metronidazole, secnidazole and hemezole were less active. Nifuroxazide, etofamide and nalidixic acid exhibited modest anti-giardial activity; quinfamide did not inhibit the growth of the parasite at a concentration of $200 \mathrm{mg} / \mathrm{L}$. Albendazole and mebendazole are promising candidates for clinical use and should be further evaluated.
\end{abstract}

\section{Introduction}

The intestinal protozoon Giardia lamblia causes a range of infection from asymptomatic carriage to persistent and severe diarrhoea with malabsorption, and the "failure to thrive" syndrome in children. ${ }^{1,2}$ This parasite occurs worldwide, although it is more frequent in developing countries. ${ }^{3}$ Mepacrine, metronidazole or furazolidone are presently recommended for treatment. ${ }^{4-6}$ Strains of $G$. lamblia obtained from different areas of the world are susceptible to these compounds, ${ }^{7-9}$ but they occasionally provoke unacceptable side effects and treatment sometimes fails, partly because the patients do not always complete the course of therapy:4-6 moreover, these drugs are active against the normal intestinal flora. ${ }^{6}$ In the present study, we tested the susceptibility of $G$. lamblia to several compounds including the benzimidazole carbamates, albendazole and mebendazole, which are currently used for the treatment of helminthiasis and which may have less effect on the bacterial flora of the gut. ${ }^{10-12} \mathrm{G}$. lamblia has been reported to be highly susceptible to albendazole and mebendazole in vitro, ${ }^{13,14}$ but the efficacy of these drugs in clinical studies is controversial. ${ }^{15-17}$ Other drugs tested included etofamide and quinfamide, which are occasionally used in the treatment of intestinal amoebiasis, ${ }^{18,19}$ nifuroxazide, a nitrofuran used in some countries for the treatment of infectious diarrhoea ${ }^{20}$ nalidixic acid, several 5-nitroimidazoles, and drugs presently used for the treatment of giardiasis.

\section{Materials and methods}

\section{Strain}

The G. lamblia strain Portland 1 (P1) was used in all experiments; it was maintained axenically at $37^{\circ} \mathrm{C}$ in Diamond's modified TYI-S-33 medium ${ }^{21}$ supplemented with heat inactivated bovine serum $10 \%$ and ceftriaxone $50 \mathrm{mg} / \mathrm{L}$. Antibiotic was omitted during experimental assays.

\section{Antimicrobial agents}

Metronidazole, tinidazole, furazolidone and mepacrine were obtained from Sigma. Other drugs were obtained in Mexico City from the manufacturers or their subsidiaries: albendazole and nifuroxazide from SmithKline-Beecham, mebendazole from Janssen, etofamide from Carlo Erba-Farmitalia, quinfamide and nalidixic acid from Winthrop, hemezole from Silanes, ornidazole from Roche, and secnidazole from Rhône Poulenc. Metronidazole, tinidazole, mepacrine and ornidazole were dissolved in water, hemezole and nalidixic acid were dissolved in sodium bicarbonate solution ( $\mathrm{pH} 7 \cdot 2)$; other compounds were dissolved in dimethylformamide.

\section{Standard growth curve}

G. lamblia trophozoites were grown axenically in Diamond's modified culture medium as described 
above; when the logarithmic phase of growth was reached, the trophozoites were detached from the substrate by cooling on water-ice for $20 \mathrm{~min}$. They were then distributed into $1.5-\mathrm{ml}$ volumes of fresh culture medium in polypropylene microcentrifuge tubes to achieve inocula ranging from $1 \times 10^{3}$ to $5.5 \times 10^{5} / \mathrm{ml}$ as determined by counting in a haemocytometer. After incubation for $48 \mathrm{~h}$ at $37^{\circ} \mathrm{C}$, the number of trophozoites was again counted and a standard curve of cell numbers versus inoculum was constructed by linear regression analysis. A growth curve was also constructed by performing daily counts over a 9-day period starting with an inoculum of $2 \times 10^{4}$ parasites $/ \mathrm{ml}$.

\section{Susceptibility assay}

Susceptibility of $G$. lamblia to the antimicrobial agents was determined by the culture method of Hill et al. ${ }^{22}$ with some modifications: serial two-fold dilutions of drugs were prepared in $1.5-\mathrm{ml}$ volumes of culture medium in microcentrifuge tubes. The tubes were then inoculated with $G$. lamblia P1 to achieve an inoculum of $5 \times 10^{4}$ trophozoites $/ \mathrm{ml}$. Controls, prepared by adding drug-free solvents to the culture media, were similarly inoculated. After incubation for $48 \mathrm{~h}$ at $37^{\circ} \mathrm{C}$, trophozoites were detached by chilling and $50 \mu \mathrm{l}$ of each culture tube was subcultured into $1.5 \mathrm{ml}$ of fresh culture medium without drug. After incubation for a further $48 \mathrm{~h}$, the final number of parasites was determined by counting in a haemocytometer. The number of viable trophozoites remaining after exposure to drug for $48 \mathrm{~h}$ was deduced from the number of trophozoites attained in the subculture by reference to the linear regression plot of cell density versus inoculum. The percentage of growth inhibition was calculated by comparison with the growth in the drug-free control cultures. The $50 \%$ inhibitory concentration (IC50) was defined as the concentration of the drug that inhibited growth by $50 \%$ as calculated by probit analysis. ${ }^{23}$ The $90 \%$ inhibitory concentration (IC90) was similarly calculated. The minimum lethal concentration (MLC) was defined as the lowest concentration of the drug at which no viable trophozoites were observed by subculture. Each experiment was done in triplicate and was repeated at least four times.

\section{Results}

\section{Growth curve}

The logarithmic phase of growth was reached within $48 \mathrm{~h}$ and the stationary phase at 5 days. The final cell density was $(1 \cdot 5-2) \times 10^{6}$ trophozoites/ml (fig. 1$)$.

\section{Linear regression analysis of growth}

Fig. 2 shows the standard curve, obtained by regression analysis, of the number of trophozoites present after incubation for $\mathbf{4 8} \mathrm{h}$ of different inocula of G. lamblia P1.
Inhibitory and lethal concentrations of antimicrobial agents

The table shows the IC50, IC90 and MLC of each of the antimicrobial agents studied. Albendazole exhibited the greatest inhibitory and lethal activity against G. lamblia $\mathrm{P} 1$, with an IC50 of $0.01 \mathrm{mg} / \mathrm{L}$ and an MLC of $0.03-0.04 \mathrm{mg} / \mathrm{L}$. The IC50 for mebendazole was $0.06 \mathrm{mg} / \mathrm{L}$ and the MLC was $0.2-0.4 \mathrm{mg} / \mathrm{L}$. Ornidazole, tinidazole and metronidazole were the most active of the 5-nitroimidazoles; secnidazole showed an intermediate activity and hemezole was the least active compound of this group. Among the antimicrobial agents currently used for the treatment of giardiasis, metronidazole was the most active, followed by mepacrine and furazolidone. Nifuroxazide, etophamide and nalidixic acid showed a modest

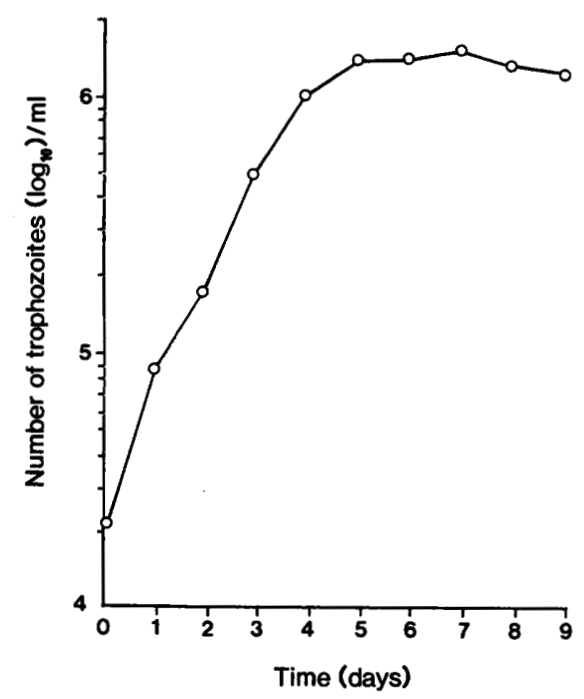

Fig. 1. Growth curve of G. lamblia P1 in Diamond's modified culture medium.

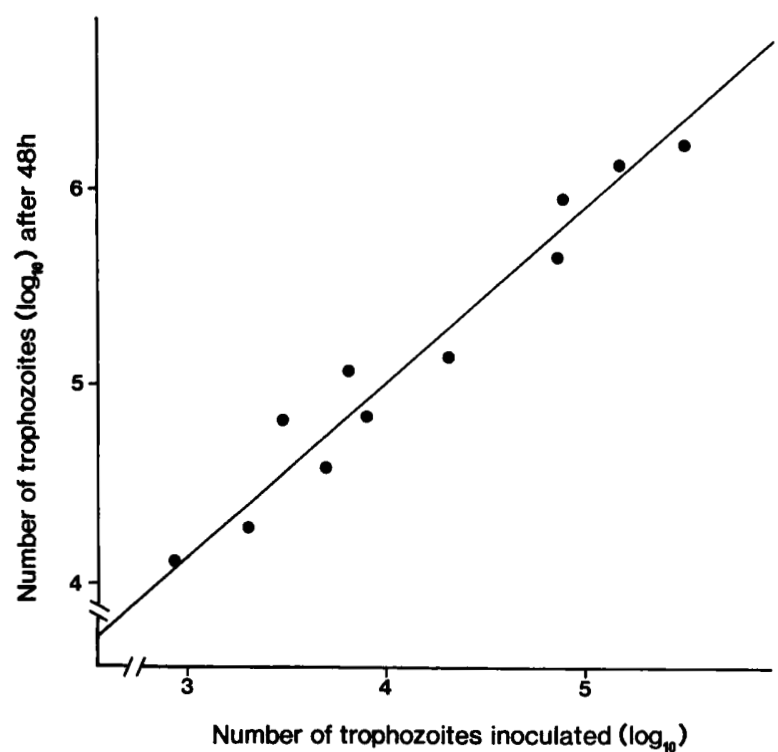

Fig. 2. Standard curve obtained by growth of different inocula of $G$ lamblia P1 for $48 \mathrm{~h}$. The curve was constructed by linear regression analysis of the results of four separate experiments, each performed in triplicate. This standard curve was used to deduce the number of viable trophozoites remaining after exposure to antimicrobial agents (see Materials and methods). 
Table. Susceptibility of $G$. lamblia to 13 antimicrobial agents

\begin{tabular}{|c|c|c|c|c|c|}
\hline $\begin{array}{l}\text { Antimicrobial } \\
\text { agent }\end{array}$ & $\begin{array}{c}\mathrm{IC50} \\
(\mathrm{mg} / \mathrm{L})\end{array}$ & SD & $\begin{array}{c}\mathrm{IC} 90 \\
(\mathrm{mg} / \mathrm{L})\end{array}$ & SD & $\begin{array}{c}\text { MLC } \\
(\mathrm{mg} / \mathrm{L})\end{array}$ \\
\hline Albendazole & 0.01 & 0.001 & 0.02 & 0.002 & $0.03-0.04$ \\
\hline Mebendazole & 0.06 & 0.020 & 0.13 & 0.030 & $0.20-0.50$ \\
\hline Ornidazole & 0.12 & 0.030 & 0.39 & 0.040 & $0.50-1.00$ \\
\hline Tinidazole & 0.14 & 0.027 & 0.44 & 0.090 & $0.50-1.00$ \\
\hline Metronidazole & 0.21 & 0.030 & 1.28 & 0.200 & $1.00-5.00$ \\
\hline Mepacrine & 0.39 & 0.050 & 1.72 & 0.350 & $1.50-5.00$ \\
\hline Furazolidone & 0.62 & 0.015 & $2 \cdot 10$ & 0.150 & $5.00-10.00$ \\
\hline Secnidazole & 0.62 & 0.140 & $2 \cdot 10$ & 0.300 & $5.00-10.00$ \\
\hline Hemezole & 1.55 & 0.440 & 3.80 & 0.500 & $10 \cdot 00-15 \cdot 00$ \\
\hline Nifuroxazide & 3.84 & 1.000 & $12 \cdot 25$ & 2.500 & $10 \cdot 00-20 \cdot 00$ \\
\hline Etofamide & 5.98 & 1.400 & 16.00 & $2 \cdot 200$ & $40 \cdot 00-50.00$ \\
\hline Nalidixic acid & $12 \cdot 13$ & 1.860 & $30 \cdot 20$ & 5.500 & $45 \cdot 00-50 \cdot 00$ \\
\hline Quinfamide & $>200$ & $\ldots$ & $\ldots$ & $\ldots$ & ... \\
\hline
\end{tabular}

IC50, 50\% inhibitory concentration; IC $90,90 \%$ inhibitory concentration; MLC, minimum lethal concentration; SD, standard deviation.

antiparasitic activity; quinfamide did not inhibit the growth of G. lamblia P1.

\section{Discussion}

These results indicate that the benzimidazole carbamates, albendazole and mebendazole are considerably more active against $G$. lamblia P1 than the other test drugs, including the 5-nitroimidazoles ornidazole, tinidazole and metronidazole. Meloni et al ${ }^{13}$ found that albendazole was 5-10 times more active than metronidazole or tinidazole against $G$. lamblia as judged by the IC50. We found the difference in activity to be somewhat greater than this, although the difference in lethal activity of these compounds was similar to that reported by Meloni et al. ${ }^{13}$ In contrast to our results, Edlin et al.$^{14}$ reported that albendazole was only 1.5 times as active as mebendazole but 50 times as active as metronidazole. The IC50 and MLC of albendazole observed in the present study were lower than those reported previously. ${ }^{13,14}$ These differences could be due to the use of different strains of $G$. lamblia and to the use of different methods to determine antimicrobial activity. The subculture method we used measures the capacity of cells to replicate (as the $\left[\mathrm{H}^{3}\right]$ thymidine uptake method also does) and this provides a more sensitive assay. ${ }^{8,22}$ Moreover, dimethyl sulphoxide (DMSO) was used to dissolve the benzimidazole carbamates in previous studies. ${ }^{13,14}$ DMSO is known to stabilise microtubules and this could alter the results of the experiments since tubulin is the target of benzimidazoles. ${ }^{12,24}$

Our results and those of others ${ }^{13,14}$ indicate that

\section{References}

1. Craft FC. Giardia and giardiasis in childhood. Pediatr Infect Dis 1982; 1: 192-211. albendazole and mebendazole might be useful alternative drugs in the treatment of giardiasis. These agents exhibit high activity against $G$. lamblia in vitro, elicit few side effects when used as anthelminthic agents and should not affect the intestinal flora. However, the results of clinical trials are conflicting. There have been three small, uncontrolled clinical studies of benzimidazoles in the treatment of giardiasis; in two of them albendazole and mebendazole were apparently curative, ${ }^{15,16}$ but in the other, mebendazole failed to eradicate the parasite. ${ }^{17} \mathrm{We}$ are performing a controlled clinical trial to evaluate the efficacy of these drugs in giardiasis.

Among 5-nitroimidazoles, ornidazole was the most effective, followed by tinidazole and metronidazole. Secnidazole, which possesses a longer half-life, ${ }^{25}$ was five-fold less active than ornidazole. Similar results were obtained by Boreham et al. ${ }^{26}$ by use of a radiometric assay. Hemezole, a compound synthesised in Mexico, was the least active of the nitroimidazoles tested. Although nifuroxazide, etofamide and nalidixic acid showed only intermediate activity, it is possible that these drugs could reach inhibitory concentrations in the lumen of the intestine at therapeutic doses. Quinfamide did not inhibit the growth of $G$. lamblia even at a concentration of $200 \mathrm{mg} / \mathrm{L}$.

Many antimicrobial agents inhibit the growth of $G$. lamblia in vitro; some of them, especially albendazole, mebendazole, but also etofamide and nifuroxazide, may be useful in treatment because of their activity and poor intestinal absorption.

This study was supported in part by a grant from the MacArthur Foundation.
2. Meyer EA, Jarroll EL. Giardiasis. Am J Epidemiol 1980; 111 : 1-12.

3. Walsh JA. Estimating the burden of illness in the tropics. In: Warren KS, Mahmoud AAF (eds) Tropical and geo- 
graphic medicine. New York, McGraw-Hill. 1984: 10731085.

4. Kavousi S. Giardiasis in infancy and childhood: a prospective study of 160 cases with comparison of quinacrine (Atabrine $^{\circledR}$ ) and metronidazole (Flagyl ${ }^{\star}$ ). Am J Trop Med Hyg $1979 ; 28$ : 19-23.

5. Levi GC, Armando de Avila C, Neto VA. Efficacy of various drugs for treatment of giardiasis. A comparative study. Am $J$ Trop Med Hyg 1977; 26: 564-565.

6. Davidson RA. Treatment of giardiasis: the North American perspective. In: Meyer EA (ed) Giardiasis. Amsterdam, Elsevier Science Publishers B V. 1990: 325-334.

7. Gordts B, Hemelhof W, Asselman C, Butzler J-P. In-vitro susceptibilities of 25 Giardia lamblia isolates of human origin to six commonly used antiprotozoal agents. Antimicrob Agents Chemother 1985; 28: 378-380.

8. Boreham PFL, Phillips RE, Shepherd RW. The sensitivity of Giardia intestinalis to drugs in vitro. J Antimicrob Chemother $1984 ; 14$ : 449-461.

9. Gillin FD, Diamond LS. Inhibition of clonal growth of Giardia lamblia and Entamoeba histolytica by metronidazole, quinacrine and other antimicrobial agents. J Antimicrob Chemother 1981 ; 8: 305-316.

10. Horton RJ. Benzimidazoles in a wormy world. Parasitol Today $1990 ; 6: 106$.

11. James DM, Gilles HM. Human antiparasitic drugs: pharmacology and usage. Chichester, John Wiley and Sons. 1985: 215.

12. Lacey E. Mode of action of benzimidazoles. Parasitol Today 1990; 6: 112-115.

13. Meloni BP, Thompson RCA, Reynoldson JA, Seville P. Albendazole: a more effective antigiardial agent in vitro than metronidazole or tinidazole. Trans $R$ Soc Trop Med Hyg 1990; 84: 375-379.

14. Edlind TD, Hang TL, Chakraborty PR. Activity of the anthelmintic benzimidazoles against Giardia lamblia in vitro. J Infect Dis 1990; 162: 1408-1411.

\section{BOOKS RECEIVED}

\section{Medical Virology 10}

Edited by L. M. De La Maza and Ellena M. Peterson. 1991. ISBN 0-306-44010-5. Plenum Press, New York. Pp. 302. $\$ 79.50$.

This book outlines the proceedings of the 10th International Symposium on Medical Virology, held in October, 1990 at Newport Beach, California. The text consists of 12 extensive reviews, presumably given as invited lectures during plenary sessions of the meeting. These are followed by 21 single-page abstracts. Although the reviews stand as individual articles, it is possible to discern some linking themes between them. Thus, the first four chapters cover various aspects of laboratory diagnostic virology, including the usefulness and applications of electronmicroscopy, the use of the polymerase chain reaction in detecting viral nucleic acid sequences, and the $Q$ replicase amplification technique. The final four chapters are concerned with aspects of prevention and treatment of virus infections, specifically those transmitted by transfusion, a novel approach to influenza vaccination by use of a purified neuraminidase subunit vaccine, lessons learned from use of an attenuated varicella vaccine, and the potential of antisense oligonucleotides as antiviral agents. The intervening third of the book is more of a mixed bag, covering determinants of measles mortality, the epidemiology of HIV-1 infection,
15. Al-Waili NS, Al-Waili BH, Saloom KY. Therapeutic use of mebendazole in giardial infections. Trans $R$ Soc Trop Med Hyg 1988; 82 : 438 .

16. Zhong HL, Cao WJ, Rossignol JF et al. Albendazole in nematode, cestode, trematode and protozoan (Giardia) infections. Chin Med J 1986; 99: 912-915.

17. Gascon J, Moreno A, Valls ME, Miró JM, Corachán M. Failure of mebendazole treatment in Giardia lamblia infection. Trans $R$ Soc Trop Med Hyg 1989; 83: 647.

18. Biagi $F$, Alvarez $R$, González $C$. Antiamoebic action of etophamide in children. Trans $R$ Soc Trop Med Hyg 1974; 68: 368-369.

19. Slighter RG, Yarinsky A, Drobeck HP, Bailey DM. Activity of quinfamide against natural infections of Entamoeba criteci in hamsters: a new potent agent for intestinal amoebiasis. Parasitology 1980; 81: 157-168.

20. Bourée P, Kouchner G, Ponti M. Double-blind study of traveller's diarrhoea using nifuroxazide. Trans $R$ Soc Trop Med Hyg 1987; 81 : 859.

21. Keister DB. Axenic culture of Giardia lamblia in TYI-S-33 medium supplemented with bile. Trans $R$ Soc Trop Med Hyg 1983; 77: 487-488.

22. Hill DR, Pohl R, Pearson RD. Giardia lamblia: a culture method for determining parasite viability. Am J Trop Med Hyg 1986; 35: 1129-1133.

23. Finney DL. Probit analysis. New York, Cambridge University Press. 1977: 20.

24. Lacey E. The role of the cytoskeletal protein, tubulin, in the mode of action and mechanism of drug resistance to benzimidazoles. Int J Parasitol 1988; 18: 885-936.

25. Videau D, Niel G, Siboulet A, Catalan F. Secnidazole. A 5nitroimidazole derivative with a long half-life. $\mathrm{Br} J$ Vener Dis 1978; 54: 77-80.

26. Boreham PF, Phillips RE, Shepherd RW : A comparison of the in-vitro activity of some 5-nitroimidazoles and other compounds against Giardia intestinalis. $J$ Antimicrob Chemother 1985; 16: 589-595.

human herpesvirus type 6 , and papillomaviruses and human cancer. Perhaps a unique feature, for a book of this nature, is the absence of a chapter on hepatitis $C$ virus, or, indeed, any of the hepatitis viruses. Paradoxically, this avoidance of such a high profile area of clinical virology may be seen as a strength, as it allows attention to be devoted to some of the "less glamorous" topics in clinical virology.

The reviews are authoritatively written and up-to-date, with several references from 1990 . Individual preferences will no doubt vary, but for this reader the chapters on HHV6, and the influenza and varicella vaccines were of most interest. Each review is followed by a question-and-answer style discussion, written in the vernacular, of perhaps limited usefulness. Similarly, the abstracts at the end of the book, mostly concerned with comparisons of diagnostic techniques, will not be of general interest. Given the need to publish these reviews with sufficient speed to ensure that the information is still relevant, one can forgive the rather large number of typographical errors. Despite these limitations, overall there is much here to hold the attention of any medical virologist, or physician with an interest in virus infections, although at the price indicated, I suspect that most copies will be purchased for library collections rather than individual use.

W. L. IRVING 